\title{
The $\mathrm{L}_{\mathrm{p}}$-mixed quermassintegrals for $0<p<1^{*}$
}

Chang-Jian Zhao

Department of Mathematics, China Jiliang University, Hangzhou 310018, P. R. China email: chjzhao@163.com
Mihály Bencze

Hărmanului street 6, 505600

Săcele-Négyfalu, Braşov, Romania email: benczemihaly@yahoo.com

\begin{abstract}
In the paper, $\mathrm{L}_{\mathrm{p}}$-harmonic addition, $\mathrm{p}$-harmonic Blaschke addition and $\mathrm{L}_{\mathrm{p}}$-dual mixed volume are improved. A new $\mathrm{p}$-harmonic Blaschke mixed quermassintegral is introduced. The relationship between p-harmonic Blaschke mixed volume and $L_{p}$-dual mixed volume is shown.
\end{abstract}

\section{Notation and preliminaries}

The setting for this paper is $n$-dimensional Euclidean space $\mathbb{R}^{n}$. Let $\mathcal{K}^{n}$ denote the subset of all convex bodies (compact, convex subsets with non-empty interiors) in $\mathbb{R}^{n}$. We reserve the letter $u$ for unit vectors, and the letter $B$ is reserved for the unit ball centered at the origin. The surface of $B$ is $S^{n-1}$. We write $V(K)$ for the (n-dimensional) Lebesgue measure of $K$ and call this the volume of $K$. Associated with a compact subset $K$ of $\mathbb{R}^{n}$, which is starshaped with respect to the origin and contains the origin, its radial function is $\rho(K, \cdot): S^{n-1} \rightarrow[0, \infty)$, defined by (see e. g. [1] and [2] )

$$
\rho(K, u)=\max \{\lambda \geq 0: \lambda u \in K\} .
$$

2010 Mathematics Subject Classification: 52A30, 46E30

Key words and phrases: p-radial addtion, p-harmonic addition, harmonic Blaschke addition, $\mathrm{p}$-harmonic Blaschke addition, $\mathrm{L}_{\mathrm{p}}$-dual mixed volume

* Research is supported by National Natural Science Foundation of China (11371334, 10971205). 
If $\rho(\mathrm{K}, \cdot)$ is positive and continuous, $\mathrm{K}$ will be called a star body. Let $\mathcal{S}^{\mathrm{n}}$ denote the set of star bodies in $\mathbb{R}^{n}$. We write $S(K)$ for the surface area of star body $K$. If $k>0$, then for all $u \in \mathbb{R}^{n} \backslash\{0\}$

$$
\rho(k K, u)=k \rho(K, u) .
$$

Let $\tilde{\delta}$ denote the radial Hausdorff metric, as follows, if $K, \mathrm{~L} \in \mathcal{S}^{\mathrm{n}}$, then (see e. g. [1])

$$
\tilde{\delta}(K, L)=|\rho(K, u)-\rho(L, u)|_{\infty}
$$

\subsection{Dual mixed volume}

The radial Minkowski linear combination, $\lambda_{1} \mathrm{~K}_{1} \widetilde{+} \cdots \widetilde{+} \lambda_{\mathrm{r}} \mathrm{K}_{\mathrm{r}}$, defined by (see [3])

$$
\lambda_{1} K_{1} \tilde{+} \cdots \tilde{+} \lambda_{r} K_{r}=\left\{\lambda_{1} x_{1} \tilde{+} \cdots \widetilde{+} \lambda_{r} x_{r}: x_{i} \in K_{i}, i=1, \ldots, r\right\},
$$

for $\mathrm{K}_{1}, \ldots, \mathrm{K}_{\mathrm{r}} \in \mathcal{S}^{\mathrm{n}}$ and $\lambda_{1}, \ldots, \lambda_{\mathrm{r}} \in \mathbb{R}$. It has the following important property:

$$
\rho(\lambda \mathrm{K} \tilde{+} \mu \mathrm{L}, \cdot)=\lambda \rho(\mathrm{K}, \cdot)+\mu \rho(\mathrm{L}, \cdot),
$$

for $K, L \in \mathcal{S}^{n}$ and $\lambda, \mu \geq 0$.

If $K_{i} \in \mathcal{S}^{n}(i=1,2, \ldots, r)$ and $\lambda_{i}(i=1,2, \ldots, r)$ are nonnegative real numbers, then of fundamental importance is the fact that the dual volume of $\lambda_{1} \mathrm{~K}_{1} \widetilde{+} \cdots \widetilde{+} \lambda_{\mathrm{r}} \mathrm{K}_{\mathrm{r}}$ is a homogeneous polynomial in the $\lambda_{i}$ given by (see e. g. [3])

$$
V\left(\lambda_{1} K_{1} \widetilde{+} \cdots \widetilde{+} \lambda_{r} K_{r}\right)=\sum_{i_{1}, \ldots, i_{n}} \lambda_{i_{1}} \ldots \lambda_{i_{n}} \widetilde{V}_{i_{1} \ldots i_{n}}
$$

where the sum is taken over all $n$-tuples $\left(i_{1}, \ldots, i_{n}\right)$ of positive integers not exceeding $r$. The coefficient $V_{i_{1} \ldots i_{n}}$ depends only on the bodies $K_{i_{1}}, \ldots, K_{i_{n}}$ and is uniquely determined by (2), it is called the dual mixed volume of $\mathrm{K}_{i_{1}}, \ldots, \mathrm{K}_{\mathrm{i}_{n}}$, and is written as $\mathrm{V}\left(\mathrm{K}_{i_{1}}, \ldots, \mathrm{K}_{\mathrm{i}_{n}}\right)$. Let $\mathrm{K}_{1}=\ldots=\mathrm{K}_{\mathrm{n}-\mathrm{i}}=\mathrm{K}$ and $K_{n-i+1}=\ldots=K_{n}=L$, then the mixed volume $\widetilde{V}\left(K_{1} \ldots K_{n}\right)$ is written as $\widetilde{V}_{i}(K, L)$. If $K_{1}=\cdots=K_{n-i}=K, K_{n-i+1}=\cdots=K_{n}=B$, then the mixed volumes $V_{i}(K, B)$ is written as $\widetilde{W}_{i}(K)$ and is called the dual quermassintegral of star body $K$ and $(n-i) \widetilde{W}_{i+1}$ is written as $S_{i}(K)$ and called the mixed surface area of $\mathrm{K}$. The dual quermassintegral of star body $\mathrm{K}$, defined as an integral by (see [4])

$$
\widetilde{W}_{i}(K)=\frac{1}{n} \int_{S^{n-1}} \rho(K, u)^{n-i} d S(u) .
$$


It is convenient to write relation (2) in the form (see [5, p.137])

$$
\begin{aligned}
& \widetilde{V}\left(\lambda_{1} K_{1} \widetilde{+} \cdots \widetilde{+} \lambda_{s} K_{s}\right) \\
& =\sum_{p_{1}+\cdots+p_{r}=n} \sum_{1 \leq i_{1}<\cdots<i_{r} \leq s} \frac{n !}{p_{1} ! \cdots p_{r} !} \lambda_{i_{1}}^{p_{1}} \cdots \lambda_{i_{r}}^{p_{r}} \widetilde{V}(\underbrace{K_{i_{1}}, \ldots, K_{i_{1}}}_{p_{1}}, \ldots, \underbrace{K_{i_{r}}, \ldots, K_{i_{r}}}_{p_{r}}) .
\end{aligned}
$$

Let $s=2, \lambda_{1}=1, K_{1}=K, K_{2}=B$, we have

$$
V(K \widetilde{+} \lambda B)=\sum_{i=0}^{n}\left(n_{i}^{n}\right) \lambda^{i} \widetilde{W}_{i}(K),
$$

known as formula "Steiner decomposition". Moreover, for star bodies K and L, (4) can show the following special case:

$$
\widetilde{W}_{i}(K \widetilde{+} \lambda L)=\sum_{j=0}^{n-i}\left(n_{j}^{n-i}\right) \lambda^{j} \widetilde{V}(\underbrace{K, \ldots, K}_{n-i-j}, \underbrace{B, \ldots, B}_{i}, \underbrace{L, \ldots, L}_{j}) .
$$

\subsection{The p-radial addition and p-dual mixed volume}

For any $p \neq 0$, the $p$-radial addition $K \widetilde{+}_{p} L$ defined by (see [6] and [7])

$$
\rho\left(K \tilde{+}_{p} L, u\right)^{p}=\rho(K, u)^{p}+\rho(L, u)^{p},
$$

for $u \in S^{n-1}$ and $K, L \in \mathcal{S}^{n}$. When $p=\infty$ or $-\infty$, the $p$-radial addition is interpreted as $\rho\left(\mathrm{K} \widetilde{+}_{\infty} \mathrm{L}, \mathrm{u}\right)=\mathrm{K} \cup \mathrm{L}$ or $\rho\left(\mathrm{K} \tilde{+}_{-\infty} \mathrm{L}, \mathrm{u}\right)=\mathrm{K} \cap \mathrm{L}$ (see e. g. [8]).

The following result follows immediately from (6).

$$
\frac{p}{n} \lim _{\varepsilon \rightarrow 0^{+}} \frac{V\left(K \widetilde{+}_{p} \varepsilon \cdot L\right)-V(L)}{\varepsilon}=\frac{1}{n} \int_{S^{n-1}} \rho(K . u)^{n-p} \rho(L . u)^{p} d S(u) .
$$

Let $K, L \in \mathcal{S}^{n}$ and $p \neq 0$, the $p$-dual mixed volume of star $K$ and $L, \widetilde{V}_{p}(K, L)$, defined by

$$
\widetilde{V}_{p}(K, L)=\frac{1}{n} \int_{S^{n-1}} \rho(K \cdot u)^{n-p} \rho(L . u)^{p} d S(u) .
$$

The Minkowski inequality for the p-radial addition stated that: If $K, \mathrm{~L} \in \mathcal{S}^{\mathrm{n}}$ and $0<\mathrm{p} \leq \mathrm{n}$, then (see [7])

$$
\widetilde{\mathrm{V}}_{\mathrm{p}}(\mathrm{K}, \mathrm{L})^{\mathrm{n}} \leq \mathrm{V}(\mathrm{K})^{\mathrm{n}-\mathrm{p}} \mathrm{V}(\mathrm{L})^{\mathrm{p}}
$$

with equality if and only if $\mathrm{K}$ and $\mathrm{L}$ are dilates.

The inequality is reversed for $p>n$ or $p<0$ 


\section{The $L_{p}$-dual mixed volume for $0<p<1$}

For $p \geq 1$, Lutwak defined the $\mathrm{L}_{\mathrm{p}}$-harmonic addition of star bodies $\mathrm{K}$ and $\mathrm{L}$, $\mathrm{K} \check{+}_{p} \varepsilon \diamond \mathrm{L}$, defined by (see [9])

$$
\rho\left(\mathrm{K} \check{+}_{\mathrm{p}} \varepsilon \diamond \mathrm{L}, \cdot\right)^{-\mathrm{p}}=\rho(\mathrm{K}, \cdot)^{-\mathrm{p}}+\varepsilon \rho(\mathrm{L}, \cdot)^{-\mathrm{p}} .
$$

As defined in $(9), K \check{+}_{p} \varepsilon \diamond \mathrm{L}$ has a constant coefficient $\mathrm{p}$ restricted to $\mathrm{p} \geq 1$. We now extend the definition so that $\mathrm{K} \check{+}_{p} \mathrm{~L}$ is defined for $0<\mathrm{p}<1$.

Definition 1 (The $\mathrm{L}_{\mathrm{p}}$-harmonic addition for $0<\mathrm{p}<1$ ) If $\mathrm{K}, \mathrm{L} \in \mathcal{S}^{\mathrm{n}}$ and $0<\mathrm{p}<1$, the $\mathrm{L}_{\mathrm{p}}$-harmonic addition of star bodies $\mathrm{K}$ and $\mathrm{L}, \mathrm{K} \check{+}_{p} \varepsilon \diamond \mathrm{L}$, defined by

$$
\rho\left(\mathrm{K} \check{+}_{\mathrm{p}} \varepsilon \diamond \mathrm{L}, \cdot\right)^{-\mathrm{p}}=\rho(\mathrm{K}, \cdot)^{-\mathrm{p}}+\varepsilon \rho(\mathrm{L}, \cdot)^{-\mathrm{p}} .
$$

From (10), it is easy that for $0<\mathrm{p}<1$ (and $\mathrm{p} \geq 1$ )

$$
-\frac{p}{n} \lim _{\varepsilon \rightarrow 0^{+}} \frac{V\left(K \check{+}_{p} \varepsilon \diamond L\right)-V(K)}{\varepsilon}=\frac{1}{n} \int_{S^{n-1}} \rho(K, u)^{n+p} \rho(L, u)^{-p} d S(u) .
$$

Definition 2 If $\mathrm{K}, \mathrm{L} \in \mathcal{S}^{\mathrm{n}}$ and $0<\mathrm{p}<1$, the $\mathrm{L}_{\mathrm{p}}$-dual mixed quermassintegral of $\mathrm{K}$ and $\mathrm{L}, \widetilde{\mathrm{V}}_{-\mathrm{p}}(\mathrm{K}, \mathrm{L})$, defined by

$$
\widetilde{V}_{-p}(K, L):=\frac{1}{n} \int_{S^{n-1}} \rho(K, u)^{n+p} \rho(L, u)^{-p} d S(u) .
$$

Theorem 1 ( $\mathrm{L}_{\mathrm{p}}$-Minkowski inequality) If $\mathrm{K}, \mathrm{L} \in \mathcal{S}^{\mathrm{n}}$ and $0<\mathrm{p}<1$, then

$$
\widetilde{\mathrm{V}}_{-\mathrm{p}}(\mathrm{K}, \mathrm{L})^{\mathrm{n}} \geq \mathrm{V}(\mathrm{K})^{\mathrm{n}+\mathrm{p}} \mathrm{V}(\mathrm{L})^{-\mathrm{p}},
$$

with equality if and only if $\mathrm{K}$ and $\mathrm{L}$ are dilates.

Proof. This integral representation (11) and together with Hölder integral inequality, this yields (12).

The case $p \geq 1$, please see literatures [10] and [11].

Theorem 2 ( $\mathrm{L}_{\mathrm{p}}$-Brunn-Minkowski inequality) If $\mathrm{K}, \mathrm{L} \in \mathcal{S}^{\mathrm{n}}$ and $0<\mathrm{p}<1$, then

$$
\widetilde{\mathrm{V}}\left(\mathrm{K} \check{+}_{\mathrm{p}} \varepsilon \diamond \mathrm{L}\right)^{-\mathrm{p} / \mathrm{n}} \geq \mathrm{V}(\mathrm{K})^{-\mathrm{p} / \mathrm{n}}+\mathrm{V}(\mathrm{L})^{-\mathrm{p} / \mathrm{n}},
$$

with equality if and only if $\mathrm{K}$ and $\mathrm{L}$ are dilates.

Proof. This follows immediately from (10) and (12). 


\section{The p-harmonic Blaschke addition for $0<p<1$}

Let us recall the concept, the harmonic Blaschke addition, defined by Lutwak [12]. Suppose $K$ and $L$ are star bodies in $\mathbb{R}^{n}$, the harmonic Blaschke linear addition, $\mathrm{K} \widehat{+} \mathrm{L}$, by

$$
\frac{\rho(\mathrm{K} \hat{+} \mathrm{L}, \cdot)^{\mathrm{n}+1}}{\mathrm{~V}(\mathrm{~K} \hat{+} \mathrm{L})}=\frac{\rho(\mathrm{K}, \cdot)^{\mathrm{n}+1}}{\mathrm{~V}(\mathrm{~K})}+\frac{\rho(\mathrm{L}, \cdot)^{\mathrm{n}+1}}{\mathrm{~V}(\mathrm{~L})} .
$$

Lutwak's Brunn-Minkowski inequality for the harmonic Blaschke addition was established (see [12]). If $K, L \in \mathcal{S}^{n}$, then

$$
\mathrm{V}(\mathrm{K} \hat{+} \mathrm{L})^{1 / n} \geq \mathrm{V}(\mathrm{K})^{1 / n}+\mathrm{V}(\mathrm{L})^{1 / \mathrm{n}},
$$

with equality if and only if $K$ and $L$ are dilates. More generally, for any $p \geq 1$, the $p$-harmonic Blaschke addition $\mathrm{K} \widehat{+}_{p} \mathrm{~L}$ defined by (see [13] and [14]).

$$
\frac{\rho\left(K \widehat{+}_{p} L, \cdot\right)^{n+p}}{V\left(K \widehat{+}_{p} L\right)}=\frac{\rho(K, \cdot)^{n+p}}{V(K)}+\frac{\rho(L, \cdot)^{n+p}}{V(L)} .
$$

The $\mathrm{L}_{p}$ Brunn-Minkowski inequality for the $p$-harmonic Blaschke addition was established ( see [13]). If $K, L \in \mathcal{S}^{n}$ and $p \geq 1$, then

$$
\mathrm{V}\left(\mathrm{K} \widehat{+}_{\mathrm{p}} \mathrm{L}\right)^{\mathrm{p} / \mathrm{n}} \geq \mathrm{V}(\mathrm{K})^{\mathrm{p} / \mathrm{n}}+\mathrm{V}(\mathrm{L})^{\mathrm{p} / \mathrm{n}},
$$

with equality if and only if $\mathrm{K}$ and $\mathrm{L}$ are dilates.

As defined in (16), $\mathrm{K} \widehat{+}_{p} \mathrm{~L}$ has a constant coefficient $\mathrm{p}$ restricted to $\mathrm{p} \geq 1$. We now extend the definition so that $\mathrm{K} \widehat{+}_{p} \mathrm{~L}$ is defined for $0<\mathrm{p}<1$.

Definition 3 (The $\mathrm{p}$-harmonic Blaschke addition for $0<\mathrm{p}<1$ ) If $\mathrm{K}, \mathrm{L} \in$ $\mathcal{S}^{\mathrm{n}}, \mathrm{O} \leq \mathrm{i}<\mathrm{n}$ and $\mathrm{O}<\mathrm{p}<1$, the $\mathrm{p}$-harmonic Blaschke addition of $\mathrm{K}$ and $\mathrm{L}$, $\mathrm{K} \widehat{+}_{\mathrm{p}} \mathrm{L}$, defined by

$$
\frac{\rho\left(\mathrm{K} \widehat{+}_{p} \mathrm{~L}, \cdot\right)^{\mathrm{n}-\mathrm{i}+\mathrm{p}}}{\widetilde{W}_{i}\left(\mathrm{~K} \widehat{+}_{p} \mathrm{~L}\right)}=\frac{\rho(\mathrm{K}, \cdot)^{n-i+p}}{\widetilde{W}_{i}(\mathrm{~K})}+\frac{\rho(\mathrm{L}, \cdot)^{\mathrm{n}-\mathrm{i}+\mathrm{p}}}{\widetilde{W}_{i}(\mathrm{~L})} .
$$

Obviously, the case $i=0$ and $p \geq 1$, is just (16), and the case of $p=1$ and $i=0$, is just (14).

Definition 4 Let $\mathrm{K}, \mathrm{L} \in \mathcal{S}^{\mathrm{n}}, 0 \leq \mathrm{i}<\mathrm{n}, 0<\mathrm{p}<1$, and $\alpha, \beta \geq 0$ (not both zero), the $\mathrm{p}$-harmonic Blaschke liner combination of $\mathrm{K}$ and $\mathrm{L}, \alpha \triangleleft \widehat{\mathrm{r}}_{\mathrm{p}} \beta \downarrow \mathrm{L}$, defined by

$$
\frac{\rho\left(\alpha \downarrow \widehat{+}_{p} \beta \downarrow L, u\right)^{n-i+p}}{\widetilde{W}_{i}\left(\alpha \widehat{K}_{p} \beta \downarrow L\right)}=\alpha \frac{\rho(K, u)^{n-i+p}}{\widetilde{W}_{i}(K)}+\beta \frac{\rho(L, u)^{n-i+p}}{\widetilde{W}_{i}(L)} .
$$


From (19) with $\beta=0$ and (1), it is easy that

$$
\frac{\rho(\alpha \triangleleft K, u)^{n-i+p}}{\widetilde{W}_{i}(\alpha K)}=\alpha \frac{\rho(K, u)^{n-i+p}}{\widetilde{W}_{i}(K)}=\frac{\rho\left(\alpha^{1 / p} K, u\right)^{n-i+p}}{\widetilde{W}_{i}\left(\alpha^{1 / p} K\right)} .
$$

Hence

$$
\alpha \triangleleft \mathrm{K}=\alpha^{1 / \mathrm{p}} \mathrm{K} .
$$

\section{Inequalities for $p$-harmonic Blaschke mixed quer- massintegral for $0<p<1$}

In order to define the $p$-harmonic Blaschke mixed quermassintegral for $0<$ $p<1$ with respect to $p$-harmonic Blaschke addition, we need the following lemmas.

Lemma 1 ([15] and [16, p.51]) If $\mathrm{a}, \mathrm{b} \geq 0$ and $\lambda \geq 1$, then

$$
a^{\lambda}+b^{\lambda} \leq(a+b)^{\lambda} \leq 2^{\lambda-1}\left(a^{\lambda}+b^{\lambda}\right) .
$$

Lemma 2 Let $0<\mathrm{p}<1,0 \leq \mathrm{i}<\mathrm{n}$ and $\varepsilon>0$. If $\mathrm{K}, \mathrm{L} \in \mathcal{S}^{\mathrm{n}}$, then

$$
\begin{aligned}
& \lim _{\varepsilon \rightarrow 0^{+}} \frac{\rho\left(K \widehat{+}_{p} \varepsilon-L, u\right)^{n-i}-\rho(K, u)^{n-i}}{\varepsilon} \\
& \geq \frac{n-i}{n-i+p}\left(\frac{S_{i}(K)}{\widetilde{W}_{i}(K)} \rho(K, u)^{n-i}+\frac{\widetilde{W}_{i}(K)}{\widetilde{W}_{i}(L)} \rho(K, u)^{-p} \rho(L, u)^{n-i+p}\right) .
\end{aligned}
$$

Proof. From (19) and in view of the L'Hôpital's rule, we obtain

$$
\begin{aligned}
& \lim _{\varepsilon \rightarrow 0^{+}} \frac{\rho\left(K \widehat{+}_{p} \varepsilon-u, u\right)^{n-i}-\rho(K, u)^{n-i}}{\varepsilon} \\
& =\lim _{\varepsilon \rightarrow 0^{+}} \frac{\left(\left(\frac{\rho(K, u)^{n-i+p}}{\widetilde{W}_{i}(K)}+\varepsilon \frac{\rho(L, u)^{n-i+p}}{\widetilde{W}_{i}(L)}\right) \widetilde{W}_{i}\left(K \widehat{+}_{p} \varepsilon L\right)\right)^{n-i /(n-i+p)}-\rho(K, u)^{n-i}}{\varepsilon} \\
& =\lim _{\varepsilon \rightarrow 0^{+}} \frac{n-i}{n-i+p}\left(\left(\frac{\rho(K, u)^{n-i+p}}{\widetilde{W}_{i}(K)}+\varepsilon \frac{\rho(L, u)^{n-i+p}}{\widetilde{W}_{i}(L)}\right) \widetilde{W}_{i}\left(K \widehat{+}_{p} \varepsilon L\right)\right)^{-p /(n-i+p)} \\
& \times\left(\widetilde{W}_{i}\left(K \widehat{+}_{p} \varepsilon-L\right)^{\prime}\left(\frac{\rho(K, u)^{n-i+p}}{\widetilde{W}_{i}(K)}+\varepsilon \frac{\rho(L, u)^{n-i+p}}{\widetilde{W}_{i}(L)}\right)+\widetilde{W}_{i}\left(K \widehat{+}_{p} \varepsilon L\right) \frac{\rho(L, u)^{n-i+p}}{\widetilde{W}_{i}(L)}\right) .
\end{aligned}
$$


In the following, we estimate the value of the derivative $\widetilde{W}_{i}\left(K \widehat{+}_{p} \varepsilon L\right)^{\prime}$. Let $f_{i}(t)=\widetilde{W}_{i}\left(K \hat{+}_{p} t-L\right)$ and from (5), (20) and (21), we obtain

$$
\begin{aligned}
f_{i}(t+\varepsilon) & =\widetilde{W}_{i}\left(K \widehat{+}_{p}(t+\varepsilon) B\right) \\
& =\widetilde{W}_{i}\left(K \widehat{+}_{p}(t+\varepsilon)^{1 / p} B\right) \\
& \geq \widetilde{W}_{i}\left(K \widehat{+}_{p}\left(t^{1 / p}+\varepsilon^{1 / p}\right) B\right) \\
& \geq \widetilde{W}_{i}\left(\left(K \widehat{+}_{p} t B\right)+\varepsilon B\right) \\
& =\sum_{j=0}^{n-i}\left({ }_{j}^{n-i}\right) \varepsilon^{j} \tilde{W}_{i+j}\left(K \widehat{+}_{p} t B\right) \\
& =f_{i}(t)+\varepsilon(n-i) \tilde{W}_{i+1}\left(K \widehat{+}_{p} t B B\right)+o\left(\varepsilon^{2}\right) .
\end{aligned}
$$

Further

$$
V\left(K \widehat{+}_{p} t-L\right)^{\prime}=\lim _{\varepsilon \rightarrow 0^{+}} \frac{f(t+\varepsilon)-f(t)}{\varepsilon} \geq(n-i) \tilde{W}_{i+1}\left(K \widehat{+}_{p} t \diamond B\right) .
$$

From $(23)$ and $(24)$ and in view of $(n-\mathfrak{i}) \tilde{W}_{\mathfrak{i}+1}(K)=S_{\mathfrak{i}}(K)$, we obtain

$$
\begin{aligned}
\lim _{\varepsilon \rightarrow 0^{+}} & \frac{\rho\left(K \widehat{+}_{p} \varepsilon L, u\right)^{n-i}-\rho(K, u)^{n-i}}{\varepsilon} \\
& \geq \frac{n-i}{n-i+p}\left(\frac{S_{i}(K)}{\widetilde{W}_{i}(K)} \rho(K, u)^{n-i}+\frac{\widetilde{W}_{i}(K)}{\widetilde{W}_{i}(L)} \rho(K, u)^{-p} \rho(L, u)^{n-i+p}\right) .
\end{aligned}
$$

Theorem 3 Let $0<\mathrm{p}<1,0 \leq \mathrm{i}<\mathrm{n}$ and $\varepsilon>0$. If $\mathrm{K}, \mathrm{L} \in \mathcal{S}^{\mathrm{n}}$, then

$$
\begin{aligned}
& \frac{n-i+p}{n-i} \lim _{\varepsilon \rightarrow 0^{+}} \frac{\widetilde{W}_{i}\left(K \widehat{+}_{p} \varepsilon L, u\right)-\widetilde{W}_{i}(K)}{\varepsilon} \\
& \geq\left(S_{i}(K)+\frac{\widetilde{W}_{i}(K)}{\widetilde{W}_{i}(L)} \frac{1}{n} \int_{S^{n-1}} \rho(K, u)^{-p} \rho(L, u)^{n-i+p} d S(u)\right) .
\end{aligned}
$$

Proof. This follows immediately from Lemma 2 and (3).

Definition 5 Let $\mathrm{K}, \mathrm{L} \in \mathcal{S}^{\mathrm{n}}, 0 \leq \mathrm{i}<\mathrm{n}$ and $0<\mathrm{p}<1$, we define the $\mathrm{p}$-ith harmonic Blaschke mixed quermassintegral of star bodies $\mathrm{K}$ and $\mathrm{L}$, denoted by $\widehat{\mathrm{W}}_{\mathrm{p}, \mathrm{i}}(\mathrm{K}, \mathrm{L})$, defined by

$$
\widehat{W}_{p, i}(K, L)=\frac{n-i+p}{n-i} \lim _{\varepsilon \rightarrow 0^{+}} \frac{\widetilde{W}_{i}\left(K \widehat{+}_{p} \varepsilon L, u\right)-\widetilde{W}_{i}(K)}{\varepsilon} .
$$


When $\mathrm{i}=0$, the $\mathrm{p}$-harmonic Blaschke mixed quermassintegral $\widehat{\mathrm{W}}_{\mathrm{p}, \mathrm{i}}(\mathrm{K}, \mathrm{L})$ becomes the $\mathrm{p}$-harmonic Blaschke mixed volume $\widehat{\mathrm{V}}_{\mathrm{p}}(\mathrm{K}, \mathrm{L})$ and

$$
\widehat{V}_{p}(K, L)=\frac{n+p}{n} \lim _{\varepsilon \rightarrow 0^{+}} \frac{V\left(K \widehat{+}_{p} \varepsilon L, u\right)^{n}-V(K)^{n}}{\varepsilon} .
$$

Theorem 4 ( $\mathrm{L}_{\mathrm{p}}$-Minkowski type inequality) If $\mathrm{K}, \mathrm{L} \in \mathcal{S}^{\mathrm{n}}, 0 \leq \mathrm{i}<\mathrm{n}$ and $0<p<1$, then

$$
\left(\widehat{W}_{p, i}(K, L)-S_{i}(K)\right)^{n-i} \geq \widetilde{W}_{i}(K)^{n-i-p} \widetilde{W}_{i}(L)^{p}
$$

Proof. This follows immediately from Theorem 3, (27) and Hölder integral inequality.

Corollary 1 If $\mathrm{K}, \mathrm{L} \in \mathcal{S}^{\mathrm{n}}$ and $0<\mathrm{p}<1$, then

$$
\left(\widehat{V}_{p}(K, L)-S(K)\right)^{n} \geq V(K)^{n-p} V(L)^{p} .
$$

Proof. This follows immediately from Theorem 4 with $i=0$.

\section{The relationship between the two mixed volumes}

In the following, we give a relationship between the p-harmonic Blaschke mixed volume $\widehat{V}_{p}(K, L)$ and the $L_{p}$-dual mixed volume $\widetilde{V}_{-p}(K, L)$.

Theorem 5 If $\mathrm{K}, \mathrm{L} \in \mathcal{S}^{\mathrm{n}}$ and $0<\mathrm{p}<1$, then

$$
\frac{\widehat{V}_{p}(K, L)}{V(K)} \geq \frac{\widetilde{V}_{-p}(L, K)}{V(L)}
$$

Proof. This follows immediately from (11), (27) and Theorem 3 with $i=0$.

We give also a relationship between the $p$-harmonic Blaschke mixed volume $\widehat{V}_{p}(K, L)$ and the $p$-dual mixed volume $\widetilde{V}_{p}(K, L)$.

Theorem 6 If $\mathrm{K}, \mathrm{L} \in \mathcal{S}^{\mathrm{n}}$ and $0<\mathrm{p}<1$, then

$$
\widehat{V}_{p}(K, L) \geq \widetilde{V}_{p}(K, L) \text {. }
$$


Proof. From (11), (12), (8), (25) and (27), we obtain

$$
\begin{aligned}
\widehat{V}_{p}(K, L) & \geq \frac{V(K)}{V(L)} \frac{1}{n} \int_{S^{n-1}} \rho(L, u)^{n+p} \rho(K, u)^{-p} d S(u) \\
& =\frac{V(K)}{V(L)} \tilde{V}_{-p}(L, K) \\
& \geq \frac{V(K)}{V(L)} V(L)^{(n+p) / n} V(K)^{-p / n} \\
& =V(K)^{(n-p) / n} V(L)^{p / n} \\
& \geq \tilde{V}_{p}(K, L) .
\end{aligned}
$$

Finally, we establish the Brunn-Minkowski inequality for the p-ith harmonic Blaschke addition.

Theorem 7 If $\mathrm{K}, \mathrm{L} \in \mathcal{S}^{\mathrm{n}}, 0 \leq \mathrm{i}<\mathrm{n}, 0<\mathrm{p}<1$ and $\lambda, \mu \geq 0$, then

$$
\widetilde{W}_{i}\left(\lambda \diamond K \widehat{+}_{p} \mu \downarrow L\right)^{p /(n-i)} \geq \lambda \widetilde{W}_{i}(K)^{p /(n-i)}+\mu \widetilde{W}_{i}(L)^{p /(n-i)},
$$

with equality if and only if $\mathrm{K}$ and $\mathrm{L}$ are dilates.

Proof. This follows immediately from (3), (19) and Minkowski integral inequality.

This case of $\lambda=\mu=1, p \geq 1$ and $i=0$ is just (17). This case of $p=1$, $\lambda=\mu=1$ and $i=0$ is just (15).

\section{References}

[1] R. Schneider, Convex Bodies: The Brunn-Minkowski Theory, Cambridge Univ. Press, 1993.

[2] R. J. Gardner, Geometric Tomography, Cambridge Univ. Press, New York, 1996.

[3] E. Lutwak, Intersection bodies and dual mixed volumes, Adv. Math., 71 (1988), 232-261.

[4] E. Lutwak, Dual mixed volumes, Pacific J. Math., 58 (1975), 531-538.

[5] Y. D. Burago, V. A. Zalgaller, Geometric Inequalities, Springer-Verlag, Berlin-New york, 1988. 
[6] R. J. Gardner, The Brunn-Minkowski inequality, Bull. Amer. Math. Soc., 39 (2002), 355-405.

[7] E. Grinberg, G. Zhang, Convolutions, transforms, and convex bodies, Proc. London Math. Soc,. 78 (1999), 77-115.

[8] R. J. Gardner, D. Hug, W. Weil, Operations between sets in geometry, J. Eur. Math. Soc. (JEMS), in press.

[9] E. Lutwak, The Brunn-Minkowski-Firey theory. II. Affine and geominimal surface areas. Adv. Math., 118 (1996), 244-294.

[10] E. Lutwak, D. Yang, G. Zhang, $\mathrm{L}_{p}$ affine isoperimetric inequalities, J. Differential Geom., 56 (2000),111-132.

[11] E. Lutwak, D. Yang, G. Zhang, Sharp affine $\mathrm{L}_{\mathrm{p}}$ Sobolev inequalities, J. Differential Geom., 62 (2002), 17-38.

[12] E. Lutwak, Centroid bodies and dual mixed volumes, Proc. London Math. Soc., 60(1990), 365-391.

[13] Y. B. Feng, W. D. Wang, Shephard type probems for $\mathrm{L}_{\mathrm{p}}$-centroid bodies, Math. Inqu. Appl., 17(3) (2014), 865-877.

[14] J. Yuan, L. Z. Zhao, G. S. Leng, Inequalities for Lp-centroid body, Taiwanese. J. Math., 11(5) (2007), 1315-1325.

[15] P. Chunaev, Hölder and Minkowski type inequalityies with alternating signs, J. Math. Ineq., 9(1)(2015), 61-71.

[16] R. P. Agarwal, P. Y. H. Pang, Opial inequalities with applications in differential and difference equations, Kluwer Academic Publishers, Dordrecht, Boston, London, 1995. 